\title{
The role of point-of-care ultrasound in the diagnosis of pericardial effusion: a single academic center retrospective study
}

\author{
Matthew G. Hanson ${ }^{1}$ and Barry Chan ${ }^{2^{*}}$ (1)
}

\begin{abstract}
Background: Symptomatic pericardial effusion (PCE) presents with non-specific features and are often missed on the initial physical exam, chest X-ray (CXR), and electrocardiogram (ECG). In extreme cases, misdiagnosis can evolve into decompensated cardiac tamponade, a life-threatening obstructive shock. The purpose of this study is to evaluate the impact of point-of-care ultrasound (POCUS) on the diagnosis and therapeutic intervention of clinically significant PCE.

Methods: In a retrospective chart review, we looked at all patients between 2002 and 2018 at a major Canadian academic hospital who had a pericardiocentesis for clinically significant PCE. We extracted the rate of presenting complaints, physical exam findings, X-ray findings, ECG findings, time-to-diagnosis, and time-to-pericardiocentesis and how these were impacted by POCUS.
\end{abstract}

Results: The most common presenting symptom was dyspnea (64\%) and the average systolic blood pressure (SBP) was $120 \mathrm{mmHg}$. 86\% of people presenting had an effusion $>1 \mathrm{~cm}$, and $89 \%$ were circumferential on departmental echocardiogram (ECHO) with 64\% having evidence of right atrial systolic collapse and $58 \%$ with early diastolic right ventricular collapse. The average time-to-diagnosis with POCUS was $5.9 \mathrm{~h}$ compared to $>12 \mathrm{~h}$ with other imaging including departmental ECHO. Those who had the PCE identified by POCUS had an average time-to-pericardiocentesis of $28.1 \mathrm{~h}$ compared to $>48 \mathrm{~h}$ with other diagnostic modalities.

Conclusion: POCUS expedites the diagnosis of symptomatic PCE given its non-specific clinical findings which, in turn, may accelerate the time-to-intervention.

Keywords: Pericardial effusion, Point-of-care ultrasound, Diagnosis

\section{Introduction}

Pericardial effusion (PCE) is a fluid collection in the pericardial sac which can result in life-threatening cardiac tamponade. Cardiac tamponade occurs when the intrapericardial pressure compresses the cardiac chambers resulting in impaired diastolic filling for which the right heart is most susceptible [1,2]. The fibrous pericardial sac has limited compliance; therefore, tamponade can

\footnotetext{
*Correspondence: barrytschan@gmail.com

2 Division of General Internal Medicine, Department of Medicine, Queen's University, Kingston, ON, Canada

Full list of author information is available at the end of the article
}

be precipitated when small volume of fluid accumulates quickly $[3,4]$. Alternatively, subacute accumulation can stretch the pericardium, increasing its compliance, such that higher PCE volume is required to result in tamponade $[1,2,5]$. The pericardial sac generally contains $50 \mathrm{~mL}$ of fluid, and effusion size is classified based on the pericardial separation distance during diastole with small effusion being $<10 \mathrm{~mm}(50-100 \mathrm{~mL})$, moderate effusion as $10-20 \mathrm{~mm}(100-500 \mathrm{~mL})$, and large effusion $>20 \mathrm{~mm}$ $(>500 \mathrm{~mL})[4,6,7]$.

Often patients with symptomatic PCE or tamponade present with non-specific symptoms and signs such as 
dyspnea, peripheral edema, tachycardia, and elevated jugular venous pressure (JVP) $[2,5]$. As such, bedside diagnosis is challenging given the non-specific symptomatology and variable exam findings $[3,5]$. Classically, Beck's Triad, comprising hypotension, increased JVP, and quiet/distant heart sounds, has been taught to screen for tamponade at the bedside $[2,8,9]$. However, the triad does not reliably predict the presence of tamponade or significant PCE in over $40 \%$ of cases [5]. Other clinical features classically described for PCE or tamponade include tachypnea, tachycardia, and pulsus paradoxus are non-specific [2]. For example, pulsus paradoxus may be present in patients with significant respiratory distress (asthma or chronic obstructive pulmonary disease exacerbation), or any mechanism that exaggerates the interventricular dependence. Conversely, pulsus paradoxus can be absent in patients with left ventricular hypertrophy, hypovolemia or atrial septal defects even in the presence of clinically significant PCE $[2,10,11]$. Some studies have looked at adjuncts to the physical exam including electrocardiogram (ECG) analysis, using chest X-ray (CXR), or point-of-care ultrasound (POCUS) to aid the diagnosis of PCE or cardiac tamponade $[5,8,10,12-$ 14]. ECG and CXR lack diagnostic sensitivity, whereas POCUS has been shown to be sensitive, specific, and potentially reduces the time-to-intervention $[1,10,12]$.

Echocardiography (ECHO) remains as the gold standard imaging modality to verify the presence of a PCE by demonstrating fluid collection in the pericardial space and interrogating for features of tamponade physiology such as systolic right atrial (RA) collapse, early diastolic right ventricle (RV) collapse, respiratory variations of transvalvular flow $[1,3,4,7]$.

To determine the rate of reported symptoms, physical exam findings, ECG and CXR abnormalities, we looked at all patients who underwent pericardiocentesis between 2002 and 2018 at a Canadian tertiary care hospital. Furthermore, we looked at ECHO findings, the length of time-to-diagnosis and time-to-pericardiocentesis, and how these were affected by POCUS.

\section{Methods}

\section{Study design}

This chart review was performed from 2002-2018 on patients who underwent pericardiocentesis at Kingston Health Sciences Centre, a tertiary care hospital in Kingston, Ontario, Canada. Ethical consent was obtained from the Queen's University Health Sciences \& Affiliated Teaching Hospitals Research Ethics Board. Patient electronic medical records were searched for the terms "pericardiocentesis + pericardial tamponade", "pericardiocentesis + cardiac tamponade", and "pericardiocentesis + pericardial effusion".
Patient records were reviewed for the presenting symptomatology, physical examination findings, CXR and ECG reports, POCUS use, time-to-diagnosis, and timeto-pericardiocentesis where available. Demographic data including age, gender, past medical history, and medications were, also, obtained where available.

In terms of POCUS resource at our institution, the prevalence of trained POCUS users in 2002 is unknown though its use was limited to Emergency Medicine. By 2018, 100\% of the Emergency Physicians were POCUS users with the majority trained via the Emergency Department ECHO (EDE) 1 program; and 30\% of General Internal Medicine were POCUS users with training ranging from part of their subspecialty fellowship program to proprietary training courses such as the Canadian Point of Care Ultrasound Society (CPOCUS). With regard to POCUS machinery, the first mention of the use of POCUS in our study was 2007, at the time, there was a total of 3 POCUS machines all of which were situated in the emergency room. As of 2018, there were 9 machines (1 for Cardiology, 2 for Critical Care, 3 for Emergency Medicine, 3 for General Internal Medicine).

As there is no POCUS archiving and reporting system locally, the beside sonographic findings and interpretation were extracted from written documentation (paper chart or electronic medical record). The CXR and ECG interpretations were derived from the finalized reports of the respective radiologist and cardiologist.

The time-to-diagnosis interval was computed from the time of presentation to the earliest mention of the presence of a PCE for a respective diagnostic modality. The time-to-pericardiocentesis interval was computed from the time of presentation to the earliest mention of the procedure being commenced or underway. Patients requiring more than one pericardiocentesis during a given visit only had the first procedure considered for the study. The differences in the means of the presenting hemodynamic profiles between the groups that was first diagnosed via POCUS and departmental ECHO were analyzed with the Welch's $t$-test with the threshold $\mathrm{p}$-value adjusted through the Bonferroni correction.

Data are reported as an overall average of all charts and include those where data were unavailable in the denominator unless otherwise specified. Statistical analysis was performed with Statistical Package for Social Sciences (SPSS) Version 26.

\section{Results}

\section{Baseline characteristics}

\section{The search algorithm returned 342 unique charts}

In all comers receiving pericardiocentesis, the average age was $63.3 \pm 14$ years and males were slightly more common than females (56 vs $44 \%$, respectively). The 
most common comorbid conditions were hypertension (37\%) and malignancy (27\%) (Table 1). 79 were procedurally related PCE (23\%) with ablation for atrial fibrillation/flutter being the most common cause.

Upon review of all the charts available via the search terms which included "Pericardiocentesis", the procedures were all performed as inpatient.

\section{Most common presentation complaint, physical exam, ECG, CXR findings}

The most common presenting complaint was dyspnea which was present in $66 \%$ of patients; and $41 \%$ had elevated JVP.

Most patients had compensated initial hemodynamics where $43 \%$ had a normal heart rate and only $9.6 \%$ were hypotensive with $\mathrm{SBP} \leq 90 \mathrm{mmHg}$.

Table 1 Baseline characteristics $(n=342)$

\begin{tabular}{ll}
\hline Age (years) & $63.3 \pm 14.1$ \\
Gender & $n(\%)$ \\
Male & $193(56)$ \\
Female & $149(44)$ \\
Cardiovascular risk factors & \\
Hypertension & $127(37)$ \\
Diabetes & $65(19)$ \\
Coronary artery disease & $59(17)$ \\
Atrial fibrillation/flutter & $63(18)$ \\
Inflammatory conditions & \\
Rheumatologic conditions & $25(7)$ \\
Pericarditis & $10(3)$ \\
Malignancy & $91(27)$ \\
Recent cardiac procedure & $31(9)$ \\
Hemodynamics on presentation & \\
Systolic blood pressure (mmHg) & $121 \pm 27$ \\
Diastolic blood pressure (mmHg) & $72 \pm 14$ \\
Systolic blood pressure $\leq 90$ mmHg (n) (\%) & $33(9.6)$ \\
Heart rate (beats per minute) & $94 \pm 22$ \\
On oral anticoagulation & \\
Warfarin & \\
Direct oral anticoagulant & $26(8)$ \\
Other cardiac medications & $5(1)$ \\
ACE inhibitor/angiotensin receptor blocker & \\
Beta-blocker & \\
Dihydropyridine calcium channel blocker & \\
Non-dihydropyridine calcium channel blocker & \\
Diuretics & $29(26)$ \\
Anti-arrhythmics & $81(18)$ \\
Vasodilators & $29(8)$ \\
\hline
\end{tabular}

$47 \%$ had tachycardia on ECG at the time of presentation; and 54\% had cardiomegaly on CXR. The remainder of other clinical features, ECG, and radiographic findings are tabulated in Table 2.

\section{Modality of initial diagnosis, time-to-diagnosis, and time-to-intervention}

For non-procedurally related PCE, 35.5\% were identified by departmental ECHO, 23.3\% via POCUS, and 19.8\% through other imaging modalities for which computed tomography $(\mathrm{CT})$ scan was most common.

The time-to-diagnosis with POCUS and departmental ECHO were on average $5.9 \mathrm{~h}$ and $45.1 \mathrm{~h}$, respectively. Time-to-pericardiocentesis was $28.1 \mathrm{~h}$ in the POCUS group in comparison to $49 \mathrm{~h}$ for the departmental ECHO group (Table 3). After adjustment with the Bonferroni correction ( $p$-value threshold $<0.0167$ is considered to indicate statistical significance), there was no statistical difference between the presenting hemodynamic profile between the two groups (Table 4).

\section{Echocardiography}

Of all patients who received a pericardiocentesis and had a measured effusion pocket, $67 \%$ had a $\mathrm{PCE} \geq 2 \mathrm{~cm}, 17 \%$ were $1-2 \mathrm{~cm}$, and $3.5 \%$ with $<1 \mathrm{~cm}$. $89 \%$ had circumferential effusion (Table 5). 64\% had evidence of systolic

Table 2 Presenting symptoms, physical exam, electrocardiogram, and chest X-ray findings $(n=342)$

\begin{tabular}{lccc}
\hline & Yes $\boldsymbol{n}$ (\%) & No $\boldsymbol{n}(\%)$ & Not available $\boldsymbol{n}$ (\%) \\
\hline Presenting symptoms & & & \\
Dyspnea & $227(66)$ & $52(15)$ & $63(18)$ \\
Orthopnea & $51(15)$ & $151(44)$ & $140(41)$ \\
Chest pain & $108(32)$ & $151(44)$ & $83(24)$ \\
Presyncope & $45(13)$ & $184(54)$ & $113(33)$ \\
Syncope & $20(6)$ & $208(61)$ & $114(33)$ \\
Peripheral edema & $41(12)$ & $189(55)$ & $112(33)$ \\
Physical exam & & & \\
Respiratory distress & $67(20)$ & $183(54)$ & $220(64)$ \\
Pulsus paradoxus & $123(36)$ & $168(49)$ & $51(15)$ \\
$\quad$ (>10 mmHg) & & & \\
Muffled heart sound & $10(3)$ & $271(79)$ & $61(18)$ \\
Elevated jugular venous & $140(41)$ & $188(55)$ & $14(4)$ \\
$\quad$ pulse & & & \\
Electrocardiogram & & & \\
Sinus tachycardia & $161(47)$ & $153(45)$ & $28(8)$ \\
Low voltage & $123(36)$ & $168(49)$ & $51(15)$ \\
Alternans & $10(3)$ & $271(79)$ & $61(18)$ \\
Chest X-ray & & & \\
Cardiomegaly & $184(54)$ & $55(16)$ & $103(30)$ \\
Pulmonary edema & $15(4)$ & $227(66)$ & $100(29)$ \\
\hline & & &
\end{tabular}


Table 3 Diagnostic modality, time-to-diagnosis, and timeto-intervention (non-procedure related $n=263$ )

\begin{tabular}{lll}
\hline Initial diagnostic modality $(\boldsymbol{n})$ & $\begin{array}{l}\text { Time-to- } \\
\text { diagnosis } \\
\text { in hours }(\boldsymbol{n})\end{array}$ & $\begin{array}{l}\text { Time-to- } \\
\text { pericardiocentesis } \\
\text { in hours }(\boldsymbol{n})\end{array}$ \\
\hline Departmental ECHO (93) & $45.1(35)$ & $49.0(67)$ \\
POCUS (61) & $5.9(47)$ & $28.1(55)$ \\
Other $^{2}$ (53) & $12.0(30)$ & $56.1(45)$ \\
Not available (56) & - & - \\
\hline
\end{tabular}

a Other imaging includes $\mathrm{CT}(n=49)$, abdominal ultrasound $(n=1)$, cardiac MRI $(n=1)$, and ventilation/perfusion (VQ) scan $(n=1)$, intraoperative transesophageal ECHO $(n=1)$

right atrial (RA) collapse, and 58\% had early diastolic right ventricular (RV) collapse.

\section{Discussion}

PCE is challenging to diagnose clinically. Larose et al. noted $80 \%$ of 50 cardiac tamponade who necessitated pericardiocentesis received either the wrong diagnosis
(36\%) or not considered as part of the differential diagnosis $(44 \%)$ [15]. The most common symptom is dyspnea is non-specific for clinically significant PCE. Furthermore, many of the classically taught physical exam findings do not accurately rule in or out PCE. In comparison to a meta-analysis by Jacob et al. to our review, 54\% (33-74\%) vs $41 \%$ had elevated JVP, $22 \%$ (11-34\%) vs $15 \%$ with muffled heart sounds, and hypotension in $28 \%$ (14-39\%) vs $10 \%$, respectively [5]. The difference in the rates of hypotension could be explained by patient population selection. In the meta-analysis, the study with the highest hypotension rate $(39 \%)$, iatrogenic PCE accounted for $31 \%$ of the cases whereas our study was $23 \%$. Trauma and surgically related PCE tend to develop acute tamponade in comparison to medical etiologies. In addition, majority of the studies took place in the 1980s (1963-1997) and comparing to a more contemporary review [16] only $15 \%$ patients presenting to the emergency department with non-traumatic causes of cardiac tamponade were hypotensive on arrival which was similar with our findings. Gandhi et al. also, noted there was less hypotensive

Table 4 Comparison of hemodynamics on presentation of the POCUS and departmental ECHO groups with the Welch's t-test

\begin{tabular}{|c|c|c|c|c|}
\hline & POCUS $(n=75)$ & Departmental ECHO $(n=126)$ & Mean difference & $p$-value ${ }^{a}$ \\
\hline Mean systolic blood pressure $(\mathrm{mmHg})$ & $112(95 \%$ Cl 107-117) & $121(95 \%$ Cl 116-126) & $8.3(95 \%$ Cl 1.2-15.5) & 0.022 \\
\hline Mean diastolic blood pressure $(\mathrm{mmHg})$ & $73(95 \%$ Cl 69-77) & 73 95\% Cl 70-76) & $0.63(95 \% \mathrm{Cl}-3.6-4.9)$ & 0.77 \\
\hline Mean heart rate (beats per minute) & 99 (95\% Cl 94-104) & $94(95 \%$ Cl 82-106) & $-5.4(95 \% \mathrm{Cl}-11-0.3)$ & 0.63 \\
\hline
\end{tabular}

a With the Bonferroni adjustment, a $p$-value of $<0.0167$ is considered to indicate statistical significance

Table 5 Departmental echocardiographic findings

\begin{tabular}{|c|c|c|c|c|c|c|c|c|c|}
\hline & \multicolumn{3}{|c|}{ All $(N=342)$} & \multicolumn{3}{|c|}{ Non-procedural etiology $(N=263)$} & \multicolumn{3}{|c|}{$\begin{array}{l}\text { latrogenic/procedural etiology } \\
(N=79)\end{array}$} \\
\hline & Yes & No & Not available & Yes & No & Not available & Yes & No & Not available \\
\hline Right atrial collapse & $219(64 \%)$ & $68(20 \%)$ & $55(16 \%)$ & $187(71 \%)$ & 47 (18\%) & $29(11 \%)$ & $32(41 \%)$ & $21(27 \%)$ & $26(33 \%)$ \\
\hline Right ventricle collapse & $199(58 \%)$ & $89(26 \%)$ & $54(16 \%)$ & $164(62 \%)$ & $7(27 \%)$ & $28(11 \%)$ & $35(44 \%)$ & $18(23 \%)$ & $26(33 \%)$ \\
\hline Left atrial collapse & $10(3 \%)$ & $275(80 \%)$ & $57(17 \%)$ & $8(3 \%)$ & $225(86 \%)$ & $30(11 \%)$ & $2(3 \%)$ & $50(63 \%)$ & $27(34 \%)$ \\
\hline Left ventricle collapse & $4(1 \%)$ & $282(83 \%)$ & $56(16 \%)$ & $2(1 \%)$ & $231(89 \%)$ & $30(11 \%)$ & $2(3 \%)$ & $51(65 \%)$ & $26(33 \%)$ \\
\hline Significant respiratory variation & $98(29 \%)$ & $41(12 \%)$ & $203(59 \%)$ & $86(33 \%)$ & $33(13 \%)$ & $144(55 \%)$ & $12(15 \%)$ & $8(10 \%)$ & $59(75 \%)$ \\
\hline Mechanical alternans & $8(2 \%)$ & $36(11 \%)$ & $298(87 \%)$ & $8(3 \%)$ & $32(12 \%)$ & $223(85 \%)$ & $0(0 \%)$ & $4(5 \%)$ & 75 (95\%) \\
\hline \multicolumn{10}{|l|}{ Effusion location } \\
\hline Circumferential & 307 (90\%) & & & $255(97 \%)$ & & & $52(66 \%)$ & & \\
\hline Regional & $19(6 \%)$ & & & $6(2 \%)$ & & & $13(16 \%)$ & & \\
\hline Not available & $16(4 \%)$ & & & $2(1 \%)$ & & & $14(18 \%)$ & & \\
\hline \multicolumn{10}{|c|}{ Deepest effusion pocket (measured during diastole) } \\
\hline$<1 \mathrm{~cm}$ & $13(4 \%)$ & & & $5(2 \%)$ & & & $8(10 \%)$ & & \\
\hline $1-2 \mathrm{~cm}$ & $57(17 \%)$ & & & $41(16 \%)$ & & & $16(20 \%)$ & & \\
\hline$>2 \mathrm{~cm}$ & $228(67 \%)$ & & & $204(78 \%)$ & & & $24(30 \%)$ & & \\
\hline Not available & 33 (10\%) & & & $11(4 \%)$ & & & $22(28 \%)$ & & \\
\hline Pericardial clot & $11(3 \%)$ & & & $2(1 \%)$ & & & $9(11 \%)$ & & \\
\hline
\end{tabular}


presentation (32\% vs $15 \%$ ) comparing cardiac tamponade cohorts from 1998-1991 to 2002-2005 [19]. This could be explained by an increased index of suspicion for the condition over the past decade [19] and access to echocardiography to assist in the diagnosis [20].

In our study, many were hemodynamically compensated on presentation with fewer than $10 \%$ being hypotensive despite $84 \%$ of all cases had moderate-to-large effusions with $>50 \%$ having RA and/or RV collapse. This is in keeping with previous studies that demonstrated normotension or even hypertension in patients with subacute or chronic PCE accumulation resulting in cardiac tamponade [16-18]. These echocardiographic-to-clinical discrepancies illustrate the hemodynamic spectrum spanning from subclinical to clinically overt yet compensated to outright decompensatory shock $[11,18]$.

Given the insidious nature of PCE, evaluation with POCUS could reduce the time-to-diagnosis which had truncated by $39 \mathrm{~h}$ in our study. In terms of time-to-pericardiocentesis, our data demonstrated the POCUS group received the intervention $21 \mathrm{~h}$ earlier than the departmental ECHO group. A smaller retrospective study by Alert et al. found a similar signal whereby the POCUS arm received pericardiocentesis $59 \mathrm{~h}$ earlier than the control [12]. However, this trend towards diagnostic and interventional efficiency should be interpreted with caution. Intuitively, POCUS would readily allow significant PCE to be identified, therefore leading to earlier diagnosis and intervention if indicated. Conversely, those who are sicker may more likely receive POCUS interrogation by the concerned physician-a selection bias. In our study, however, there was no significant hemodynamic difference between the two groups on presentation. Perhaps this could be explained by an increase use of POCUS as training programs foster the skillset into their curriculums. As of 2012, 100\% of Fellow of the Royal College of Physicians of Canada-Emergency Medicine residency programs (FRCPC-EM) and $88 \%$ of the Certification in the College of Family Physicians Emergency Medicine (CCFP-EM) residency programs have formally incorporated POCUS as part of their curriculums [21]. A recent national in 2016 amongst Canadian emergency physicians demonstrated an overall increase in POCUS usage compared to 2007 [22]. Critical Care and General Internal Medicine are, also, recommending POCUS training which will foster its clinical utilization [23, 24].

This study has several limitations. First of all, it takes place at a single university hospital, therefore the results may not extrapolate to other systems where the etiological prevalence differs especially in trauma centers.

Secondly, this is a retrospective study spanning from 2002 to 2018 whereby many charts were only partially converted to the electronic version for review and, also, given the lack of systematic history and physical exam findings data documentation, and POCUS archiving system, the dataset is subjected to significant registry bias as certain elements may not have been recorded or explicitly sought. Furthermore, some of the cases were transferred from peripheral hospitals whereby the presenting complaints, vitals, and initial diagnostic tests were not available. Uncaptured PCE that was discovered by POCUS that warranted a pericardiocentesis will affect the timeto-diagnosis and time-to-pericardiocentesis aggregate estimation as well. Nevertheless, as discussed above, the rates determined from our limited study are congruent with to a large meta-analysis [5]. Also, the POCUS group's time-to-diagnosis was on average $39 \mathrm{~h}$ less than the group diagnosed via departmental echocardiography. This time margin is quite wide unlikely explainable by exclusion of undocumented POCUS alone.

This study did not include two groups: (a) those who died from cardiac tamponade as they would not have received pericardiocentesis and (b) those who were diagnosed with a PCE and discharged without a pericardiocentesis. As such, their respective time-to-diagnosis intervals are not incorporated into the calculation. However, given both groups did not receive pericardiocentesis, its exclusion will not affect the time-to-pericardiocentesis estimation. Also, the intent of the study is to investigate only those with clinically significant PCE who warranted pericardiocentesis on the same admission.

The estimation of the time-to-diagnosis and time-topericardiocentesis is dependent on timestamps from the time of initial presentation to the date and time when the procedure being performed is recorded. As such, the time frame will not be precise to the minute. Consequently, the interval is reported in hours instead which will be less error prone. However, a prospective study is necessitated to verify the value of POCUS upon these definitive endpoints and with current POCUS archiving systems the analysis can be more rigorous.

\section{Conclusion}

Clinically significant PCE presents with non-specific findings whereby a high degree of clinical suspicion is necessitated. However, POCUS is a readily available bedside diagnostic tool that can quickly rule in the diagnosis. Whether it may expedite definitive intervention, further studies would be required.

\section{Abbreviations}

DBP: Diastolic blood pressure; ECHO: Echocardiogram; ECG: Electrocardiogram; CXR: Chest X-ray; JVP: Jugular venous pulse; LA: Left atrium; LV: Left ventricle; PCE: Pericardial effusion; POCUS: Point-of-care ultrasound; RA: Right atrium; RV: Right ventricle; SBP: Systolic blood pressure; VQ: Ventilation perfusion. 


\section{Acknowledgements}

Many thanks to Dr. Christopher Smith, Division Head of General Internal Medicine at Queen's University, who has been supportive with this research endeavour.

\section{Authors' contributions}

There was no funding provided for this article. Data collection was done by $B C$, data analysis and manuscript preparation were done by MGH and BC. All authors read and approved the final manuscript.

\section{Funding}

Not applicable.

\section{Availability of data and materials}

The data compilation is available via the corresponding author.

\section{Ethics approval and consent to participate}

This study was approved by the Queen's University Health Sciences and Affiliated Teaching Hospitals Research Ethics Board.

\section{Consent for publication}

Consent for publication was approved by the Queen's University Health Sciences and Affiliated Teaching Hospital Research Ethics Board.

\section{Competing interests}

The authors declare that they have no competing interests.

\section{Author details}

1 Division of Cardiology, Department of Medicine, Queen's University, Kingston, ON, Canada. ${ }^{2}$ Division of General Internal Medicine, Department of Medicine, Queen's University, Kingston, ON, Canada.

\section{Received: 10 August 2020 Accepted: 19 January 2021}

Published online: 04 February 2021

\section{References}

1. Vakamudi S, Ho N, Cremer PC (2017) Pericardial effusions: causes, diagnosis, and management. Prog Cardiovasc Dis 59(4):380-388. https://doi. org/10.1016/j.pcad.2016.12.009

2. Roy CL, Minor MA, Brookhart MA (2007) Does this patient with a pericardial effusion have cardiac tamponade? JAMA 297(16):1810-1818. https:// doi.org/10.1001/jama.297.16.1810

3. Ceriani E, Cogliati C (2016) Update on bedside ultrasound diagnosis of pericardial effusion. Intern Emerg Med 11(3):477-480. https://doi. org/10.1007/s11739-015-1372-8

4. Hoit BD (2017) Pericardial effusion and cardiac tamponade in the new millennium. Curr Cardio Rep 19(7):57. https://doi.org/10.1007/s1188 6-017-0867-5

5. Jacob S, Sebastian JC, Cherian PK, Abraham A, John SK (2009) Pericardial effusion impending tamponade: a look beyond Beck's triad. Am J Emerg Med 27(2):216-219. https://doi.org/10.1016/j.ajem.2008.01.056

6. Azarbal A, LeWinter MM (2017) Pericardial effusion (2017). Cardiol Clin. 35(4):515-524. https://doi.org/10.1016/j.ccl.2017.07.005

7. Alerhand S, Carter JM (2019) What echocardiographic findings suggest a pericardial effusion is causing tamponade? Am J Emerg Med 37(2):321326. https://doi.org/10.1016/j.ajem.2018.11.004

8. Liu RB, Donroe JH, McNamara RL, Forman HP, Moore CL (2017) The practice and implications of finding fluid during point-of-care ultrasonography: a review. JAMA Intern Med 177(12):1818-1825. https://doi. org/10.1001/jamainternmed.2017.5048
9. Beck CS (1935) Two cardiac compression triads. JAMA 104(9):714-716. https://doi.org/10.1001/jama.1935.02760090018005

10. Argula RG, Negi SI, Banchs J, Yusuf SW (2015) Clinical investigations role of a 12-lead electrocardiogram in the diagnosis of cardiac tamponade as diagnosed by transthoracic echocardiography in patients with malignant pericardial effusion. Clin Cardio 28(3):139-144. https://doi.org/10.1002/ clc.22370

11. Argulian E, Messerli F (2013) Misconceptions and facts about pericardial effusion and tamponade. Am J Med 126(1):858-861. https://doi. org/10.1016/j.amjmed.2013.03.022

12. Alpert EA, Amit U, Guranda L, Mahagna R, Grossman SA, Bentancur A (2017) Emergency depart point-of-care ultrasonography improves time to pericardiocentesis for clinically significant effusions. Clin Exp Emerg Med. 4(3):128-132. https://doi.org/10.15441/ceem.16.169

13. Nagdev A, Stone MB (2011) Point-of-care ultrasound evaluation of pericardial effusions: does this patient have cardiac tamponade? Resuscitation 82(6):671-673. https://doi.org/10.1016/j.resuscitation.2011.02.004

14. Mathur AP, Saini A, Lucas BP et al (2013) Diagnostic accuracy retrospectively of electrocardiographic findings and cancer history for tamponade in patients determined to have pericardial effusion by transthoracic echocardiogram. Am J Cardiol 111(7):1062-1066. https:// doi.org/10.1016/j.amjcard.2012.11.064

15. Larose E, Ducharme A, Mercier LA et al (2000) Prolonged distress and clinical deterioration before pericardial drainage in patients with cardiac tamponade. Can J Cardiol 16:331-336 (PMID: 10744796)

16. Kapoor T, Locurto M, Farina GA et al (2012) Hypotension is uncommon in patients presenting to the emergency department with nontraumatic cardiac tamponade. J Emerg Med 42(2):220-226. https://doi. org/10.1016/j.jemermed.2010.05.071

17. Brown J, MacKinnon D, King A et al (1992) Elevated arterial blood pressure in cardiac tamponade. N Engl J Med 327(7):463-466. https://doi. org/10.1056/nejm199208133270704

18. Reddy PS, Curtiss El, Uretsky BF (1990) Spectrum of hemodynamic changes in cardiac tamponade. Am J Cardiol 66(20):1487-1491. https:// doi.org/10.1016/0002-9149(90)90540-h

19. Gandhi S, Scheneider A, Mohiuddin S et al (2008) Has the clinical presentation and clinician's index of suspicion of cardiac tamponade changed over the past decade. Echocardiography 25(3):237-241. https://doi.org/1 0.1111/j.1540-8175.2007.00592.x

20. Levine MJ, Lorell BH, Diver DJ et al (1991) Implications of echocardiographically assisted diagnosis of pericardial tamponade in contemporary medical patients: detection before hemodynamic embarrassment. J Am Coll Cardiol 17(1):59-65. https://doi.org/10.1016/0735-1097(91)90704-d

21. Kim DJ, Theoret J, Liao MM et al (2012) The current state of ultrasound training in Canadian emergency medicine programs: perspectives from program directors. Acad Emerg Med 19(9):e1073-e1078. https://doi.org/ 10.1111/j.1553-2712.2012.01430.x

22. Leschyna M, Hatam E, Britton S et al (2019) Current state of point-ofcare ultrasound usage in Canadian emergency departments. Cureus 11(3):e4246. https://doi.org/10.7759/cureus.4246

23. Arntfield RT, Millington SJ, Ainsworth CD et al (2014) Canadian recommendations for critical care ultrasound training and competency. Can Respir J 21(6):341-345. https://doi.org/10.1155/2014/216591

24. Ma IWY, Arishenkoff S, Wiseman J et al (2017) Internal medicine pointof-care ultrasound curriculum: consensus recommendations from the Canadian Internal Medicine Ultrasound (CIMUS) group. J Gen Intern Med 32:1052-1057. https://doi.org/10.1007/s11606-017-4071-5

\section{Publisher's Note}

Springer Nature remains neutral with regard to jurisdictional claims in published maps and institutional affiliations. 